\title{
Bericht aus dem Arbeitskreis Psychotherapie
}

Seit fünf Jahren stellen wir jährlich die Aktivitäten unseres Arbeitskreises Psychotherapie der Bundesdirektorenkonferenz in der Psychiatrischen Praxis vor. Der wiedergegründete Arbeitskreis widmet sich seit nunmehr sieben Jahren der Anwendung psychotherapeutischer Methoden im Rahmen der stationären psychiatrischen Versorgung, aber auch im Bereich von Gemeindepsychiatrie, der Tätigkeit psychiatrischer Institutsambulanzen sowie in der teilstationären Versorgung. Zu diesen Bereichen unterstützt er den Vorstand der BDK in entsprechenden psychotherapiespezifischen Fragestellungen und vertritt die Belange der Versorgungskrankenhäusern auch in der Ständigen Kommission der ärztlichen psychotherapeutischen Verbände bei der Bundesärztekammer.

Nach seiner Neugründung hat der Arbeitskreis bereits sieben bundesweite Treffen durchführen können, das geplante achte Treffen ist wie so viele andere Veranstaltungen in diesem Jahr unter den Maßgaben der Pandemie nicht möglich gewesen. Es war geplant, sich in Göttingen intensiv mit den Themen der Behandlung von Patienten mit traumatischen Erlebnissen auseinanderzusetzen. Eine „traumasensitive Psychiatrie“ sollte vorgestellt werden und Erfahrungen sowie neue therapeutische Konzepte zur Behandlung von Traumafolgestörungen sollten im Vordergrund stehen. Wir hoffen, diese Tagung zu einem späteren Zeitpunkt nachholen zu können und werden diese zentralen Fragestellungen nicht aus dem Fokus verlieren.

Unabhängig von den ausgefallenen Veranstaltungen war aber gerade die Pandemiezeit gekennzeichnet von vielen Themen, die verschiedene Aspekte psychotherapeutischer Arbeit berühren.
Ein wichtiger Aspekt war die Fortsetzung der therapeutischen Arbeit während des Lockdowns: Im ambulanten Bereich galt es, die notwendigen infektionsprophylaktischen Maßnahmen einzuhalten. Hier war es vor allem die Ausweitung von telemedizinischen Angeboten im Bereich der Richtlinienpsychotherapie, aber auch im Bereich der Arbeit psychiatrischer Institutsambulanzen. Auch konnten Patienten, die in der Pandemie ihre stationären Behandlungen verfrüht beendet haben, oft auf diese Weise nachbetreut werden. Im Bereich der stationären Behandlung gab es häufig die Notwendigkeit, aus Gründen von Abstands- und Hygieneregeln auf Gruppenpsychotherapien zu großen Teilen zu verzichten oder aber die Gruppengrößen drastisch zu reduzieren. Auch im Bereich tagesklinischer Angebote mussten zeitweilig in vielen Regionen deutliche Abstriche gemacht werden. In manchen Bundesländern ging es sogar so weit, dass Allgemeinverfügungen die vorübergehenden Schließungen von psychosomatischen und psychotherapeutischen Behandlungseinrichtungen nach sich zogen. Alle diese Themen wirkten sich selbstverständlich in unterschiedlicher Weise auf die psychotherapeutische Arbeit in den Fachkrankenhäusern für Psychiatrie, Psychotherapie und Psychosomatik aus.

Inwiefern das anstehende Jahr 2021 eine veränderte Form stationärer psychotherapeutischer Arbeit mit sich bringen wird, lässt sich aus heutiger Sicht kaum sagen. Ob die Pandemie einen Innovationsschub auslöst oder ob sie dazu führen wird, dass wir unsere teils lange und gut etablierten Therapieangebote reduzieren müssen, wird erst in einigen Jahren wirklich zu beurteilen sein. Sicher wird es von großer Bedeutung sein, in den anstehenden Diskussionen die Interessen unserer Mitarbeiterinnen und Mitarbeiter sowie vor al- lem auch unserer Patientinnen und Patienten engagiert zu vertreten!

Ungeachtet der Auswirkungen der Pandemie auf unsere klinische Arbeit hat sich leider im zurückliegenden Jahr gesundheitspolitisch ebenfalls einiges getan, das wir aus Sicht der stationären Psychotherapie intensiver hätten beleuchten wollen: So ist die Einführung der neuen Personalrichtlinie PPP-RL leider nicht ausgesetzt worden, die Diskussion der Rolle der ärztlichen wie psychologischen Psychotherapeutinnen und -therapeuten in den Kliniken konnte aber in der Pandemie nicht adäquat weitergeführt werden. Schließlich steht die Einführung der neuen Direktausbildung für nicht ärztliche Psychotherapeuten im Rahmen der entsprechenden Masterstudiengänge zum Wintersemester an. Die spätere Einbindung der Absolventen, aber auch die Beteiligung unserer Kliniken an der Direktausbildung müsste dringend weiter diskutiert werden. Hier stehen wichtige Entscheidungen an, die Gegenstand unserer nächsten Tagung im Jahr 2021 sein müssen. Sollte es erneut nicht gelingen, diese als Präsenztagung durchzuführen, werden wir wohl auf alternative Kommunikationswege zurückgreifen müssen.

Korrespondenzadresse

Prof. Dr. Andreas Schuld, M. A.

Klinikum Ingolstadt

Krumenauerstraße 25

85049 Ingolstadt

Deutschland

andreas.schuld@klinikum-ingolstadt.de 